\title{
An Affordable, Virtual Reality Based Training Application for Cardiopulmonary Resuscitation
}

\author{
H. N. Kegalle ${ }^{\# 1}$, H. S. U. Liyanage ${ }^{2}$, K. L. Jayaratne ${ }^{3}$, M. I. E. Wickramasinghe ${ }^{4}$
}

\begin{abstract}
In Medical science, proficiency in Cardiopulmonary Resuscitation (CPR) is considered as a vital skill for physicians. For training CPR, medical professionals use mechanical manikin which has some drawbacks when it comes to the realism of the simulation and the feedback of performance. This paper presents a Virtual Reality (VR) based solution to address some of these shortcomings. The approach here is augmenting the mechanical manikin with VR using HTC Vive, Leap Motion Controller, and a glove. To test the acceptance of this solution, a user-based evaluation was carried out. $85.7 \%$ of the users who have participated in the evaluation have expressed their preference upon using VR in CPR training. Even though the overall evaluation depicts a neutral output, this study opens avenues for future research in combining VR into medical training processes.
\end{abstract}

Keywords - Cardiopulmonary Resuscitation training, engineering in medicine and biology, medical simulation, virtual reality, hand occlusion

\section{INTRODUCTION}

$\mathrm{C}$ ardiopulmonary Resuscitation (CPR) is a lifesaving procedure that is considered as an essential skill for medical professionals. It prevents failure and curbing damage that may badly affect the critical organs of the human body due to the low oxygen flow [1]. CPR is conducted by compressing the chest of a patient suffering from cardiac arrest. Acquiring proper training in CPR is crucial because an untrained person might harm the patient's life.

CPR should consist of 30 compressions performed at a depth of 5-10 cm underneath the sternum at a constant rhythm with an artificial airway established through intubation [2]. Each compression also has to have a constant pressure applied through the duration of the compression. These guidelines have been set by the European Resuscitation Council (ERC).

In most countries, CPR training is carried out on mechanical manikins as they offer a good approximation of the tactile and haptic feel to the person who is administering CPR. But these manikins are not capable of giving the CPR practitioner an immersive and dynamic training. Additionally, there are more advanced manikins that are not affordable for the government-sponsored health sector of developing countries such as Sri Lanka. Therefore, integrating VR into CPR training presents an interesting path of research. Some studies have explored the potential of integrating VR into CPR training [3]-[5]. But these approaches are not suitable as the solutions are too bulky or expensive. One main objective of this study is to implement an economically viable solution while providing the user an experience to accurately train the

Correspondence: H. N. Kegalle ${ }^{\# 1}$ (E-mail: hnk@ucsc.cmb.ac.lk) Received: 13-09-2019 Revised: 01-02-2021 Accepted: 24-02-2021

H. N. Kegalle ${ }^{\# 1}$, H. S. U. Liyanage ${ }^{2}$, K. L. Jayaratne ${ }^{3}$, M. I. E. Wickramasinghe $^{4}$ are from the University of Colombo School of Computing (hnk@ucsc.cmb.ac.lk, samali.liyanage93@gmail.com, klj@ucsc.cmb.ac.lk, mie@ucsc.cmb.ac.lk). process of CPR without the aid of a trainer.

To accomplish the targets of immersive experience and commercial viability, the proposed solution is implemented with affordable, off-the-shelf hardware that is available commercially. The center of the setup is a mechanical manikin, which provides tactile feedback to the user. HTC Vive is used as the viewing device and the Leap Motion Controller (LMC) to map the user's hands into the virtual world. The pressure applied during chest compressions is measured using an arrangement of four (4) load cells [6].

A VR application containing a humanoid avatar lying in the hospital setting is created using Unity 3D. The avatar is then mapped with the mechanical manikin in the physical world. When performing chest compressions on the manikin, the user can see as the compressions are performed on the patient avatar through the Head Mounted Device (HMD) [7].

A user-based evaluation was carried out to assess the acceptance level of the proposed approach. Two user groups were created according to their prior experience on CPR. Both novice users and expert users were given the VR application and the traditional manikin, and their feedback has been collected through a questionnaire.

During the implementation process, the researchers have encountered several problems. The occlusion problem of the LMC and the requirement for the users to keep looking at the hands within the field view of the leap motion were two major limitations found.

At the posture of the user performing chest compressions, one hand of the user should be kept on the other. The researches have realized that the occluded hand of the user was not visible in the virtual environment at this posture.

The reason for this issue was the failure of the LMC to track the occluding hand. A solution for this hand occlusion problem is also presented in this paper.

The occlusion problem experienced in this study is considered as Type-I inter-object occlusion, as the occluding objects are of the same type [8]. Several studies have been addressed the problem of human hand occlusion [9]-[11]. But these proposed hand tracking methods are not acceptable to be integrated into the above-mentioned VR application, as some of the solutions are heavy and some need a large dataset in the case of training a neural network.

In this research, a light-weight glove has been introduced to track the occluded hand of the user. Finger movements of the occluding hand can also be identified with the proposed glove-based method. The integration of the implemented glove into the existing VR application is done by mapping the glove coordinates with the graphical occluded hand in the VR application.

To gauge the acceptance of the solution proposed for the occluding hand, quantitative and qualitative evaluations were conducted. The position accuracy of the glove is measured by moving the glove on a grid and the experience of the users was evaluated through a questionnaire. 
There are two main contributions of this study. The first one is the design and the implementation of a preliminary solution for CPR training using VR. The other contribution is the hand tracking glove introduced as the solution for the hand occlusion problem.

The rest of the paper is structured as follows: Section II comprises of an analysis of the related work to this study. The design overview of the proposed solution is described in Section III. Section IV will describe the overall setup of the solution and, the experiments and results will be detailed in Section V. Finally, the conclusions of the study will be detailed in Section VI along with the future work.

\section{RELATED WORK}

VR has been used in the implementation of different simulated training solutions in a variety of vocations [12]. In the field of medicine, VR has been used to train medical personnel in different procedures from simple checkups to complex surgeries [3]. The integration of VR to train CPR is one such instance where immersive [13] and non-immersive [4] solutions have been explored.

To simulate haptic and tactile feedback, either a mechanical manikin or an external force feedback machine has been used [4]. The respective studies of Semeraro et al. [14] and Pramanik and Mannivanam [13], have enhanced a mechanical manikin and relied on them for the tactile and haptic component of the setups. Further, Pramanik and Mannivanam have enhanced the manikin by adding a 'force plate' to measure the pressure applied during the compression.

Tian et al. [4] and Khanal et al. [5] have used force feedback hardware to remove the reliance on the manikin. The final goal of the VR based CPR training application proposed in this study is also to replace the mechanical manikin. But the devices used, Novint Falcon and the Omega-3 are expensive compared to the manikin and thus the cost of the final setup suggested by this study may increase.

The evaluation of Semeraro et al. [14], have focused on the user's experience while they train with the setup. The experts of CPR were the group of participants who have used the system and submitted their feedback through a questionnaire. $84.6 \%$ of the participants have had a positive attitude towards using VR in CPR. Novices in CPR were not associated with the evaluation and because of that, it was not possible to check whether there is any effect on the results, if the users had no prior experience in CPR.

A comparison on the skills acquired using the VR solution against mechanical manikin was conducted by Pramanik and Mannivanam [13]. In their research, the participants were given a score for their performance, but the scoring system has not been specified in the paper. The results of the comparison have shown that the users trained with the VR solution have acquired a higher score than the users who were trained with the manikin. This result explains the possible achievements of integrating VR into the CPR training application.

The studies mentioned above have indicated that there is a possibility to implement a CPR training application using VR. But further explorations are needed for some of the matters, such as the impact on user attitudes depending on their prior experience in CPR. This study focuses to examine those problems while providing a VR solution for CPR training.
On reviewing the body of work available for hand occlusion, it can be seen that a diversity of approaches has been taken by the researches to discover a solution.

To identify the finger movements of the occluding hand, Yamamoto et al. [15] and Baldi et al. [16] have come up with a glove implementation. The study of Yamamoto et al. has attached color markers on to the fingertips of the glove and Baldi et al. have estimated the finger movements using MARG sensor data.

Researches have made several attempts [17], [18] to explore a solution for the occlusion problem using hardware devices. The lighthouse base station of the HTC Vive system is one such device that emits Infrared Rays (IR) for object tracking in the existence of occlusion.

Quinsones et al. [18] have implemented a device called "Hive tracker" which communicates with the lighthouse base stations to perform positional tracking. According to Quinsones et al., since the implemented tracker is small in size, it can be connected to small surfaces that need to be tracked. Hive tracker provides the positional tracking ability, which this study hopes to explore. According to the average error rate specified, that may lead to an inaccurate hand tracking in this proposed study.

When it comes to the evaluation process of Quinsones et al. [18] have concentrated on tracking the path of the implemented small device. The commercial hand tracker of the Vive setup and the Hive tracker both were moved along a specific path parallel to the floor and compared their tracking ability. The evaluation fails to gauge the movement along the $\mathrm{y}$-axis to compare the accuracy in a $3 \mathrm{D}$ environment. With the evaluation conducted, the Hive tracker marks a higher average error of $10 \mathrm{~mm}$, compared to the commercial tracker.

The above approaches point out the ability to track the occluded objects and also, the channels to be explored such as providing an improved method of 3D positioning in an IR environment. This study aims at exploring the above problem while making it integrable to the VR based CPR training application.

\section{DESIGN OVERVIEW}

To accomplish the aim of enhancing the mechanical manikin with VR, a set of activities has to be addressed in the hardware aspect.

\section{A. Track the location and orientation of the manikin}

The mechanical manikin, which represents the patient is the tactile source of this application. To correctly overlay the virtual avatar on the manikin, it is a must to identify the manikin's location and the orientation within the $3 \mathrm{D}$ environment.

\section{B. Track the user's hands and map them into the virtual environment}

The hands of the user, are the main administering tool that helps in performing CPR. Therefore, the hands have to be tracked up to the finger level, and then it has to be mapped with the virtual environment.

\section{Provide the user with a view of the synthetic environment}

User should be able to immerse in the synthetic environment, to interact with the virtual avatar. In addition, 


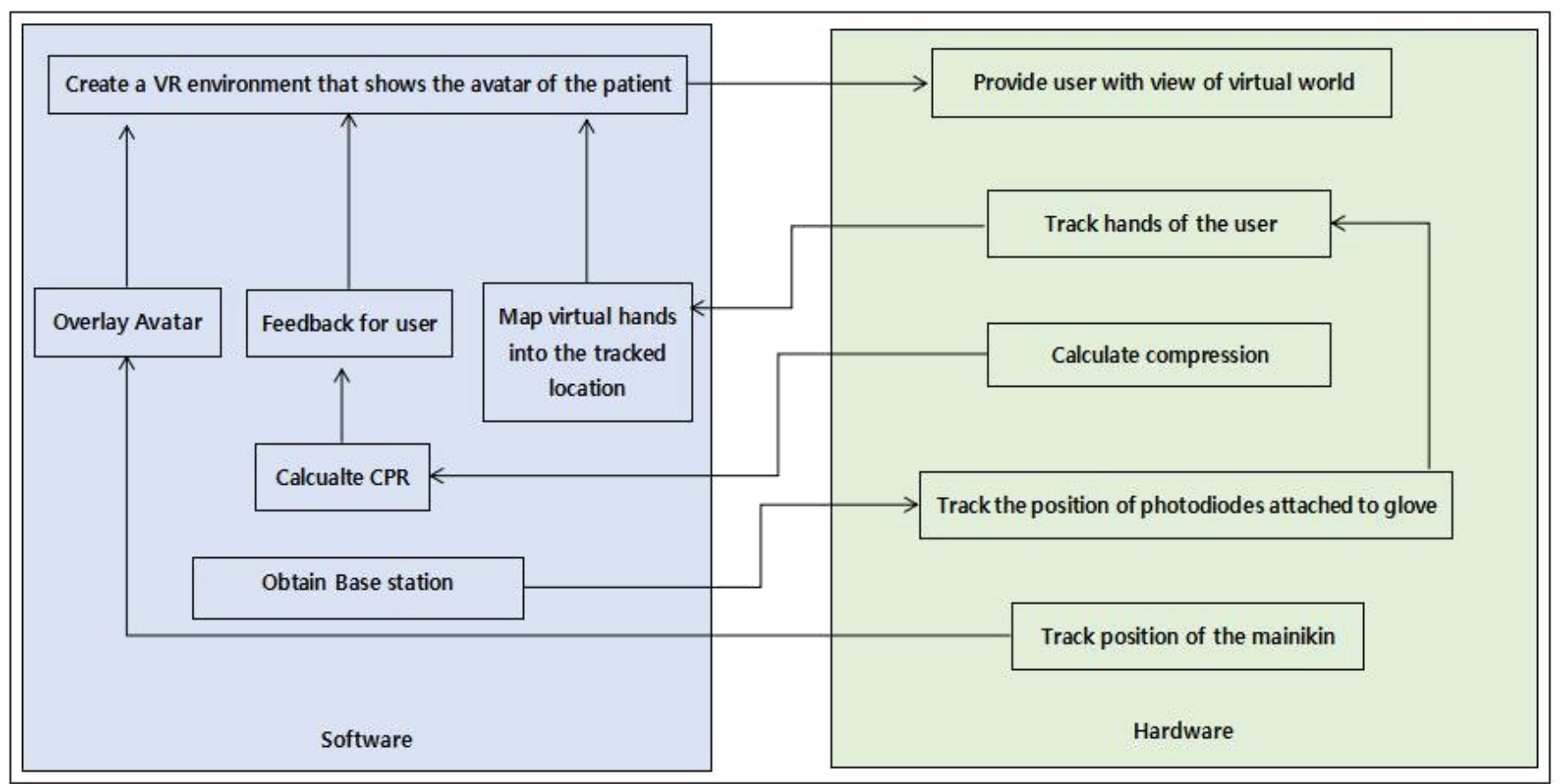

Fig. 1 Overall flow of tasks

the method uses to provide the synthetic view for the user has to be a method that does not make a disturbance in navigation.

\section{Measure the parameters of chest compressions}

The final goal of this application is to be used as a training source for the CPR trainees. Therefore, the application must be able to detect successful compressions by measuring the parameters such as the compression depth, compression count and the pressure applied.

From the software perspective, the study has to address another set of activities.

\section{E. Create a virtual world}

A virtual world that preserves the realistic condition has to be created and then, the created illusion can be projected on to the viewing device of the user.

\section{$F$. Overlay the avatar onto the manikin}

When the location and the orientation of the manikin in the 3D environment are acquired, the VR software should be able to find the corresponding coordinates in the virtual world. Then mapping the virtual avatar on the identified location has to be performed.

\section{G. Calculate compression parameters}

The parameter values mentioned in section III-D are calculated based on hardware readings. These values have to be converted into a form in which the user can identify the validity of compression by comparing the parameter values.

\section{H. Provide the user with feedback}

The user has to be provided with the feedback, based on the calculated parameters in section III-D. This feedback is crucial for the training application as the validity of the compressions are decided based on it.

According to section $\mathrm{I}$, the problem of tracking the occluded hand is an impediment that occurred in the course of the implementation. To provide a solution to that problem, the researches have used the lighthouse base stations of the HTC Vive system.

The following are the activities that have been taken place for tracking the occluded hand of the user.

\section{Obtain the base station information}

The lighthouse base station of the Vive system is a device that emits IR (Infrared) rays in a specific pattern. To identify the coordinates of the IR absorbing objects in a 3D environment, information such as the base station center coordinates and rotation matrix of the base station has to be acquired.

\section{J. Track the positions of the photodiodes}

The application should be able to track the location of moving photodiodes with the least latency. Then the values obtained have to be converted to the coordinates in the $3 \mathrm{D}$ environment.

\section{K. Map virtual hand into the tracked location}

To improve the realism of the application while providing the user with better training, the user needs to observe correct hand posture through the viewing device. Therefore, the occluded hand of the user in the real world has to be mapped with the graphical hand in the virtual world.

The flow of these tasks can be seen in Fig. 1 along with the relationships between them on the hardware and software aspect.

\section{IMPLEMENTATION}

The implementation of this study will be discussed in two stages. The initial part of this section will explain the implementation details on enhancing the mechanical manikin with VR, while the second part of this section will discuss glove implementation which is the proposed solution for the occluded hand. 
To address the design aspects mentioned under Section III, the study has used commercially available hardware such as

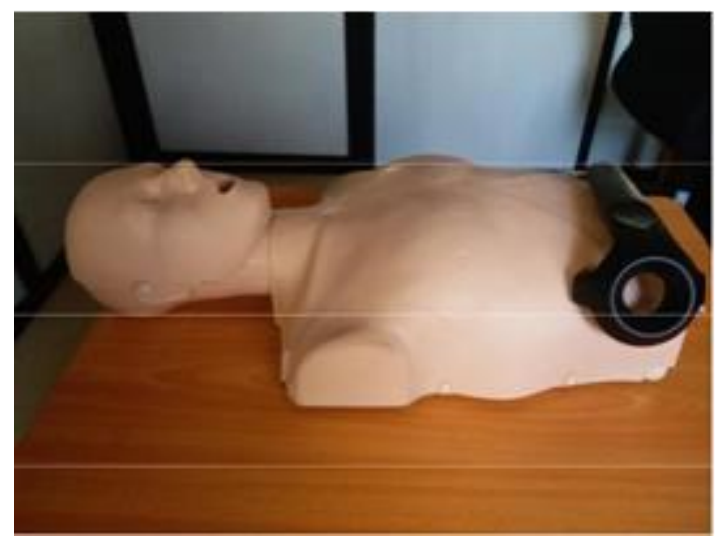

Fig. 2 Hand controller attached to the manikin

HTC Vive, Leap Motion, and Arduino board connected to a series of electronic components. Besides, free or community editions of software Unity3D and blender 7 has been used in the implementation process.

The application created using Unity3D is hosted on a PC which has the following specifications, to meet the processing needs of the LMC and the HTC Vive VR devices.

- Processor: Intel ${ }^{\circledR}$ Core i7-7700

- RAM: 8.00GB

- Graphics Card: NVIDIA GeForce GTX 1060 6GB

- Operating System: Windows 10

\section{A. Creating and viewing the virtual world}

The virtual environment consists of an avatar lying on a bed in a hospital setting is created with Unity3D. Then the view is projected to the Vive HMD. The human avatar is downloaded from mixamo.org and modified by adding new bones to the skeleton system of the avatar following the requirements. The connection between the Unity application and the HMD is created by adding Steam VR asset into the Unity project.

There are two box-like devices called base stations, which help in tracking the location of HMD and two hand controllers. Therefore, the location of the user, wearing HMD also can be tracked within the VR environment. The user's view through HMD is getting changed according to the user's head movements.

\section{B. Tracking the location of the manikin and overlaying the avatar}

To track the location of the manikin, the hand controller provided with HTC Vive is attached as seen in Fig. 2. The position of the hand controller in the real world is converted to the coordinates of the virtual world by a component in the software asset that is used to connect Vive hardware with the VR application.

The component in the above-mentioned Unity asset is then attached to the avatar that represents the patient. The positioning of the avatar concerning the position of the hand controller asset in the application is determined empirically. With this, the avatar is overlaid onto the manikin when the VR application is launched. It was detected that when performing chest compressions, as the manikin's chest moves there is a tendency for the hand controller to be moved as well. Due to this movement of the controller, the avatar also moves and makes misalignment with the manikin.

Based on the assumption that the manikin is stationary, this problem is solved by tracking the location of the manikin only once at the beginning of the simulation. After 5 seconds the avatar was decoupled from the hand controller.

\section{Tracking the user's hands and mapping them into the virtual world}

Leap Motion Controller is a device that uses IR rays to track human hands. In this stage of the study, hand tracking is done using an LMC attached in front of the Vive HMD. As discussed in Section IV-B, similar to the Vive device, LMC also has a Unity asset that converts the position of the hands in the real-world to the synthetic world coordinates.

During the implementation, it was observed that the LMC fails to track the location of the occluded hand. Following the details provided in section II, one hand of the user should be placed on top of the other, to perform chest compressions. Due to this standard posture, failure in tracking the occluded hand is inevitable.

A temporary solution for this issue was implemented with the 'collider' construct available in Unity3D. Colliders are similar to the bounding box concept [19] in Computer graphics. The colliders can be used to trigger events when entering, exiting, and staying within each other's bounds.

\section{Handling the failure to track hands due to occlusion}

Under the assumption, the left hand is occluded by the right hand, the following algorithm was used to handle the failure of hand tracking when hands occlude each other.

1: $\quad$ lh $=$ graphical left hand;

2: $\quad$ rh = graphical right hand;

3: $\quad$ lh.c $=$ collider attached to the graphical left hand;

4: $\quad$ rh.c $=$ collider attached to the graphical right hand;

5: $\quad$ copy lh;

6: while Application is running do

7: $\quad$ if lh.c and rh.c collide then

8: $\quad$ copy lh copy(lh);

9: $\quad$ attach copy lh to rh;

10: deactivate(lh);

11: else if (lh.c and hr.c do not collide) and (lh is not active) then

12: destroy (copy lh);

13: active(lh);

14: end if

15: end while

Graphical hands were attached with two separate colliders as mentioned in Lines 3 and 4. In the implementation of this algorithm, to test the condition specified in Line 7 , an event was triggered when the left hand's collider stays within the right hand's collider.

As the graphical left hand disappears from the virtual environment due to the occlusion, deactivation of the left hand mentioned in Line 10 happens automatically. Each time the condition in Line 7 is met, a copy of the graphical left hand is created and stored in the memory.

To eliminate this process of unnecessarily storing copies of the graphical left hand, the copies made are destroyed as described in Line 12. 


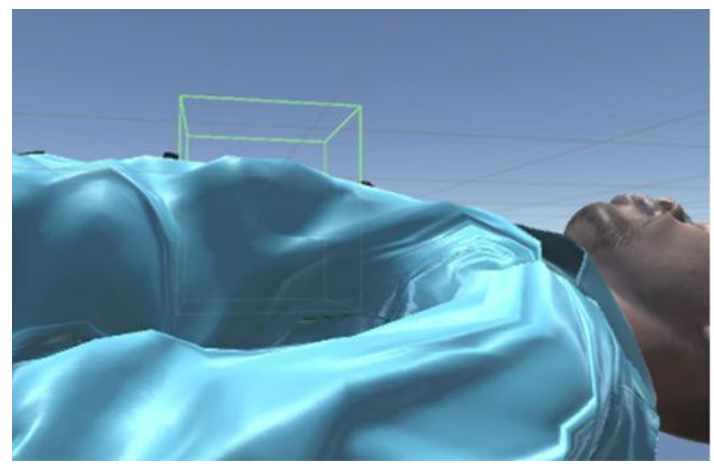

Fig. 3 Collider added to the chest area of the avatar

\section{E. Detecting compressions and measuring compression parameters}

The correct positioning of the user's hands on the patient's chest has to be identified. Then the depth of the compression has to be measured, to detect whether the compression provided by the user falls within the standard range. Additionally, the compression depth is needed to deform the avatar's chest to provide the visual experience to the user.

A collider, seen in Fig.3 is attached to the chest of the patient. When the graphical hands are entered and stays within the collider, it is decided that the user's hand have placed correctly to begin CPR.

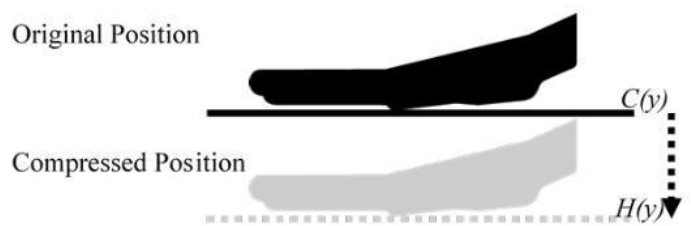

Fig. 4 Calculating compression depth

1) Measuring Compression depth: A Game object titled, "chest level" has been placed in the avatar's chest area which is taken as the marker against where the chest compressions are measured. At the start of the compression, the user's hands are placed at this level. Y-coordinates of the hand position and the chest level object are compared to calculate the compression depth as seen in Fig. 4.

The compression depth $\mathrm{D}(\mathrm{y})$ is calculated using equation 1 , where $\mathrm{C}(\mathrm{y})$ is the $\mathrm{y}$-coordinate of the chest level object and $\mathrm{H}(\mathrm{y})$ is the $\mathrm{y}$-coordinate of the hand.

$$
\mathrm{D}(\mathrm{y})=\mathrm{C}(\mathrm{y})-\mathrm{H}(\mathrm{y}) \quad \text { since } \mathrm{H}(\mathrm{y})<\mathrm{C}(\mathrm{y})
$$

2) Deforming the chest of the avatar: The bone added to the skeleton of the avatar is moved along the $y$-axis by the value of $\mathrm{D}(\mathrm{y})$ to deform the avatar's chest. If the coordinates of the new position of the bone are $\mathrm{B}^{\prime}(\mathrm{y})$ and the original position is $\mathrm{B}(\mathrm{y})$, equation 2 can be derived.

$$
\mathrm{D}(\mathrm{y})=\mathrm{B}(\mathrm{y})-\mathrm{B}^{0}(\mathrm{y}) \quad \text { since } \mathrm{B}^{0}(\mathrm{y})<\mathrm{B}(\mathrm{y})
$$

The new position of the bone according to equation 3 , can be derived using equations 1 and 2 .

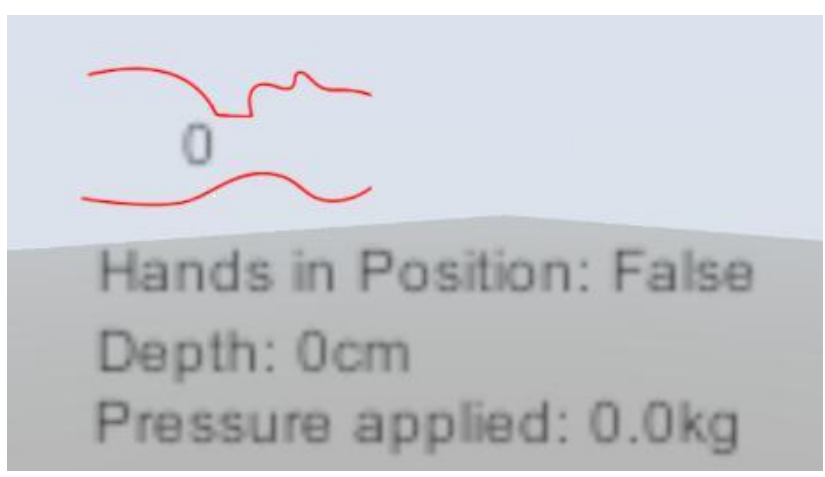

Fig. 5 Calculating compression depth

$$
\mathrm{B}^{0}(\mathrm{y})=\mathrm{B}(\mathrm{y})-\mathrm{C}(\mathrm{y})+\mathrm{H}(\mathrm{Y})
$$

In this section, the y-coordinate referred to the coordinate along the $y$-axis of the coordinate system in the Unity game engine.

3) Number of correct compressions: In this study, a compression that reaches the depth between $5 \mathrm{~cm}$ to $10 \mathrm{~cm}$ is considered as a correct compression. Therefore, when a compression meets the threshold of $5 \mathrm{~cm}$ and stays below $10 \mathrm{~cm}$, the compression count will be increased by one. If the user's hands are removed from the chest avatar's chest area, the compression count will be reverting to 0 .

4) Measuring the pressure applied: To find the pressure applied during the compression, a full-bridge load cell arrangement has been used. The arrangement is placed between the rubber casing and the inner sponge of the manikin, in the chest area where the compressions are expected to be performed.

The analog signal generated by the load-cell arrangement has been amplified using an HX711 amplifier and then connected to an Arduino Uno board. The data is streamed to the VR application hosts in the PC through the serial port.

Initially, the load-cell arrangement is calibrated using an Arduino sketch. Then another sketch is uploaded onto the board to measure, convert, and stream the applied pressure. Both these sketches were downloaded from the bogde/HX711 [20] Git repository.

\section{F. Providing the user with information and feedback}

As this is a training application, it should be able to provide information and feedback to the user on their performance of CPR. For that, the measurements and the calculations are done on various parameters of the compressions can be used. An information panel as seen in Fig. 5 is attached to a fixed point in the field view of the user.

The panel contains an outline of the human upper body, as seen in Fig.5, to indicate whether the user has placed the hands at the correct position. The outline remains red when the hands are incorrectly placed on the avatar's chest and will turn into yellow while the hands in position. When the compression depth lies between $5 \mathrm{~cm}$ to $10 \mathrm{~cm}$, the outline turns green but when it is below $5 \mathrm{~cm}$ will remain yellow and turn purple when the compression reaches beyond $10 \mathrm{~cm}$. The integer in between two lines indicates the number of correct compressions performed in one cycle [21]. 


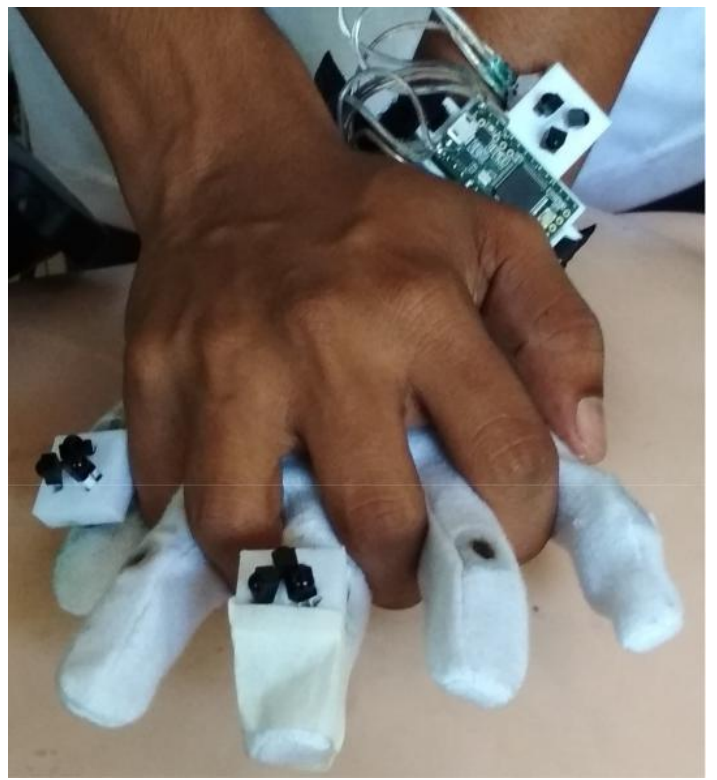

Fig. 6 Glove with three photodiode circuits

\section{G. Tracking the occluded hand using the glove}

As stated in section III - B, the hands of the user play a huge role in performing chest compressions. For a better visual experience, the user should be able to see a similar virtual view as the real world. Therefore, it is very important to display both hands in the original posture. To address this issue, a reliable solution to track the occluding hand was discovered using a glove.

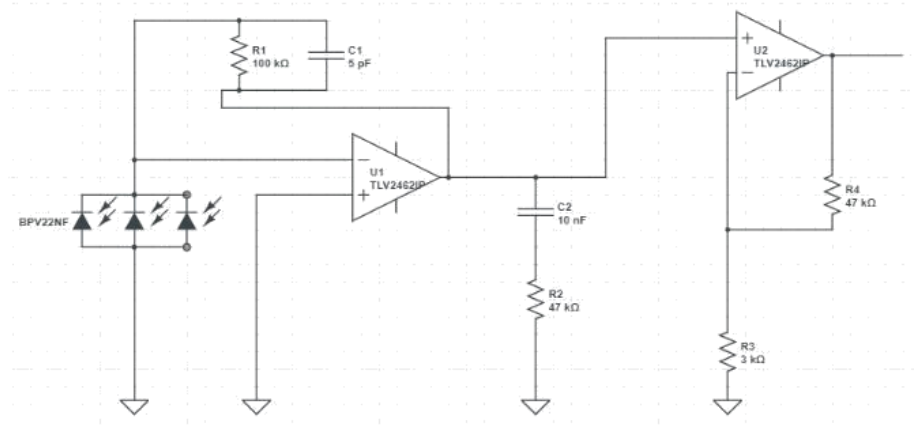

Fig. 7 Circuit Diagram for One Photodiode Circuit

1) Identifying the position of the glove: Photodiode is a device that absorbs IR and generates an electric current. In an environment where IR rays are flooding, it is possible to find the locations of photodiodes. Because the lighthouse base stations of the Vive system emits IR, a glove implemented with photodiodes can be used to track the location of the hand.

In this study, it was assumed that it is always the right hand that occludes the left. Under this assumption, a glove for the left hand, seen in Fig. 6 has been implemented using a Teensy 3.2 USB development board and a set of photodiode circuits. The processing module; the Teensy board was attached to an adjustable wrist band. Three photodiode circuits were attached to the wrist, thumb, and middle finger of the glove.

To capture IR in $360^{\circ}$, where one photodiode detects IR in a range of $120^{\circ}$, the circuit is built with three photodiodes connected in the shape of a tetrahedron. According to the circuit diagram in Fig.7 the generated signal is amplified and then connected to the Teensy development board. To accomplish the target of making the circuit small in size, the circuit was designed as a Printed Circuit Board (PCB) of size $0.8 \mathrm{~cm} \times 0.8 \mathrm{~cm}$ with Surface Mount Devices (SMD). The process of streaming data from the teensy board to the PC was done through a USB port.

The base station information mentioned in Section III- I was collected using a teensy sketch. To acquire the $\mathrm{x}, \mathrm{y}, \mathrm{z}$ coordinates of the photodiode circuit, another sketch was uploaded onto the teensy board. The codes were downloaded from the Git repository, ashtuchkin/vive-diy-position-sensor [22].

2) Mapping the occluded hand into the virtual world: A 3D hand model of the left hand has been downloaded and added into the VR application. The $\mathrm{x}, \mathrm{y}, \mathrm{z}$ coordinates collected from each sensor, which were attached to the wrist, thumb, and middle finger have been mapped with the respective parts of the 3D hand model. The positioning of the hand model in relation to the position of the sensor is determined empirically. The necessity of an additional hand tracking method occurs with the matter of two hands occlude each other. Therefore, according to the proposed solution, the 3D hand model should be inserted into the VR application only at the occurrence of occlusion. To handle this insertion of the 3D hand model, the following algorithm has been used.

1: lh=virtual left hand;

2: rh=virtual right hand;

3: lh.temp=new temporary virtual left hand;

4: while application is running do

5: if lh not visible then

6: $\quad$ make lh.temp visible;

7: end if

8: if lh visible then

9: $\quad$ make lh.temp not visible;

10: end if

11: end while

According to lines 5 and 6, when the graphical left hand created with LMC is not visible in the VR environment, the added hand model is set active. Otherwise, the $3 \mathrm{D}$ hand model is set inactive.

This solution can be extended to handle the occlusion of both hands by creating another glove which suits for the right hand.

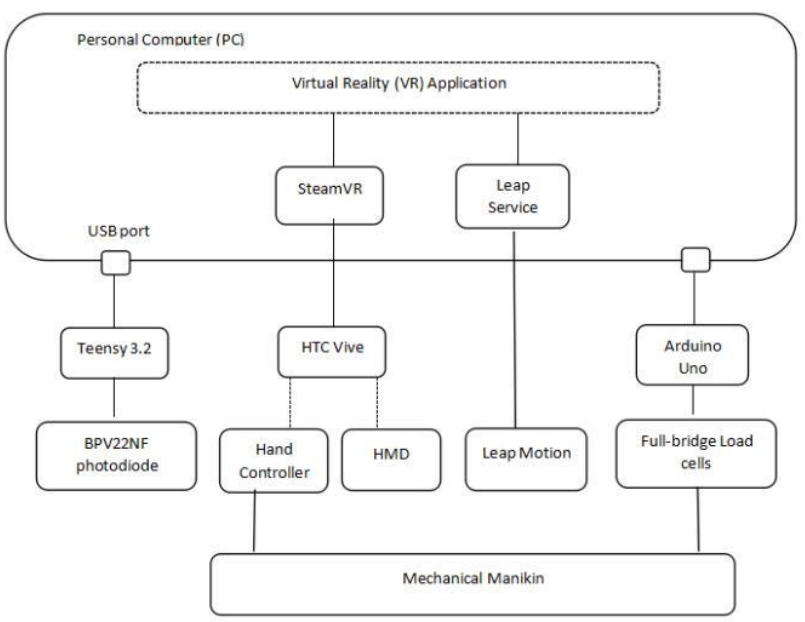

Fig. 8 Connectivity of Software and Hardware Components 


\section{H. Component connectivity}

A collection of hardware devices was used in the implementation of the VR application. Namely, HTC Vive (HMD, hand controller, base stations), leap motion controller, full-bridge load cells, Arduino Uno board, mechanical manikin, Teensy development board, photodiodes. All the devices are connected to a PC that contains the software drivers. The relationships among the components are given in Fig. 8.

\section{EVALUATION AND RESULTS}

\section{A. Acceptance of the VR application}

A user-based evaluation was conducted to determine the acceptability of the proposed VR application from the perspective of potential users. Two user groups were selected for the evaluation based on their experience in performing CPR. One group consists of assistant nurses from the Sri Lanka Navy and the Sri Lanka Fire Brigade who were proficient in CPR. The other group was novices, students, and research staff from the University of Colombo School of Computing (UCSC). The number of participants in expert and novice groups is 23 and 51 respectively. The dissimilarity of these group size is due to the difficulty of finding CPR trained participants.

In the expert group, none of the participants had experience using VR. Participants of the novice group were aware of the technology, but they had little or no experience in using VR. Therefore, it can be said that both groups had a similar experience in the case of using VR.

To remove the biases that may arise due to the exposure of one method before exposing to the other, the novice group was again divided into two subgroups (N1 and N2). One group was given the VR solution before the manikin, while the other group was given with manikin before using the VR solution. But the expert group was not divided into two subgroups, because all the participant in that group already have the experience on training CPR with the mechanical manikin. Therefore, the expert group was directly exposed to the VR solution in order to collect responses.

After carrying out CPR training with both methods for the two subgroups N1 and N2 of the novice users, feedback was collected with a survey of Likert scale questions. The points of the Likert scale arrangement can be seen in Table I.

TABLE I

LIKERT SCALE ARRANGEMENT

\begin{tabular}{|l|l|}
\hline 1 & Strongly Disagree \\
\hline 2 & Disagree \\
\hline 3 & Somewhat Disagree \\
\hline 4 & Neither Agree or Disagree \\
\hline 5 & Somewhat Agree \\
\hline 6 & Agree \\
\hline 7 & Strongly Agree \\
\hline
\end{tabular}

The 12 questions given for the users to collect feedback is given in Table II.
TABLE III

QUESTIONS USED FOR THE EVALUATION OF VR APPLICATION

\begin{tabular}{|c|c|}
\hline & Questions \\
\hline Q1 & It was difficult to wear the headset \\
\hline $\mathrm{Q} 2$ & $\begin{array}{l}\text { It has been difficult to perform chest } \\
\text { compressions using the modified manikin }\end{array}$ \\
\hline Q3 & $\begin{array}{l}\text { It has been difficult to navigate in the virtual } \\
\text { space }\end{array}$ \\
\hline Q4 & $\begin{array}{l}\text { The information panel provided useful } \\
\text { information }\end{array}$ \\
\hline Q5 & I felt the patient was really in front of me \\
\hline Q6 & $\begin{array}{l}\text { I felt my hands were aligned with the virtual } \\
\text { ones }\end{array}$ \\
\hline Q7 & $\begin{array}{l}\text { The visualization of the chest compressions felt } \\
\text { real }\end{array}$ \\
\hline Q8 & I could feel I was immersed in a 3D space \\
\hline Q9 & It was difficult to reach out and touch the patient \\
\hline $\mathrm{Q} 10$ & The interaction with the patient felt realistic \\
\hline Q11 & $\begin{array}{l}\text { The traditional manikin can be improved using } \\
\text { Virtual Reality }\end{array}$ \\
\hline Q12 & $\begin{array}{l}\text { I would like to use Virtual Reality in my CPR } \\
\text { training }\end{array}$ \\
\hline
\end{tabular}

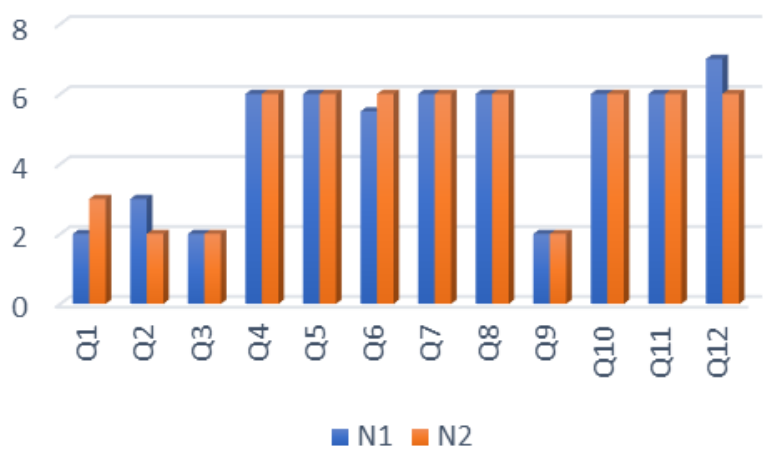

Fig. 9 Results of the user-based evaluation for novice user subgroups

Comparison of group N1 and N2 is used to detect whether there is any bias in using the VR application for CPR before the mechanical manikin and vice versa. According to figure 9 , it can be seen that there are no large distinctions between two groups on each of the statement, while slight dissimilarities can be seen regarding Q1, Q2 and Q12.

A point to be discussed from the analysis is the spread of opinion for Q1; "it was difficult to wear the headset". Spread of the median values obtained for $\mathrm{N} 1(=2)$ and $\mathrm{N} 2(=3)$ might be because some of the novice users found it difficult to put the HMD and adjust it for comfortable use.

The discrepancy in the opinion for Q2 and Q12 can be explained with a few extreme cases of responses compared to the general response in the ideas of the difficulty in performing chest compression with the modified manikin and the preference of using VR in CPR training.

Thus, it can be assumed that the two user groups (N1 and N2) can be combined and create a homogeneous group since the opinions on most of the statements are generally in the same direction. It can be also stated that the order of the method being exposed generates a little to no bias based on the observations made. 


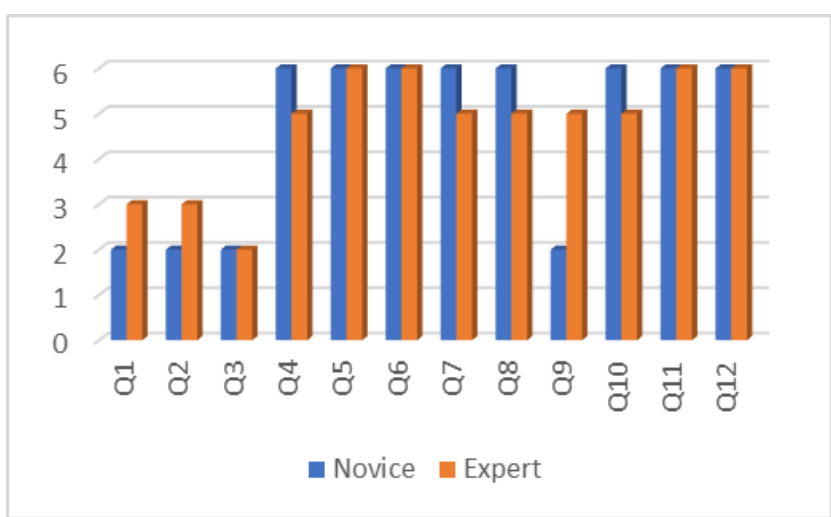

Fig. 10 Results of the Main User-based Evaluation for Novice and Expert user groups

The comparison between novice $(\mathrm{N})$ and expert (E) user groups are presented in figure 10. According to the information, a wide disagreement between the user groups can be seen in the opinion for the statement in Q9 which depicts the difficulty of reaching and touching the patient. The difference in the responses of two user groups, Novices $($ median $=2)$ disagreed while the Experts agreed (median=5) upon Q9 might be due to the difficulty of expert users to keep hands in the field view of LMC. Since the novice users were technically proficient compared to the experts, novice group had not experienced the trouble of managing hand postures to reach out the patient.

The difference in the spread of opinion between $\mathrm{N}$ (mode=6) and $\mathrm{E}$ (mode=3), for the statement in Q4 is because of the experience gap between two user groups. Most of the expert users claimed that they would prefer more advanced details to be displayed on the information panel while the novices, who had no experience in CPR, wish to have any information that will be useful for the training.

When considering the overall feedback, there are slight differences of the responses received upon several expressions when comparing the novice users and the experts. But still, the responses obtained for Q11 and Q12 illustrates the participants of both groups have agreed on using VR for CPR training with the improved manikin. The proficiency gained by an individual who is inexperienced in performing chest compressions cannot be measured by conducting an evaluation once, given that the expertise level improves gradually with the time. Therefore, it is proposed to conduct an evaluation with several iterations with the same set of users. However, the proposed approach of integrating VR into CPR as an affordable training application extends new research paths.

\section{B. Acceptance of the glove-based solution}

To examine the acceptance of the solution provided for the hand occlusion problem, quantitative evaluation, and qualitative evaluation was conducted. The objective of the quantitative evaluation is to analyze the position accuracy of the photodiode circuits. A user-based evaluation was carried out to fulfill the goal of determining the attitudes of users towards the proposed solution.

The accuracy of position tracking was inspected using a Cartesian grid of size $0.4 \mathrm{~m} \times 0.4 \mathrm{~m}$ with grid lined spaced by $10 \mathrm{~cm}$. The implemented circuit was placed on different grid points and their 3D coordinates were collected. According to the results, the average position errors are as follows:

- Average position error in $\mathrm{X}$ direction $=0.01409 \mathrm{~m}$
- Average position error in Y direction $=0.00402 \mathrm{~m}$

- Average position error in $\mathrm{Z}$ direction $=0.00431 \mathrm{~m}$

Considering the low error rates for the positions of $\mathrm{X}, \mathrm{Y}, \mathrm{Z}$ directions, it can be said that the majority of the position data given by the system is accurate. But essentially, for the circuits to be tracked, photodiodes must be exposed to the base stations.

The evaluation carried out to explore the user attitudes towards the glove-based solution was conducted with the participation of 20 students from the University of Colombo School of Computing.

The participants were divided into two groups (G1 and G2) to eliminate any bias that may occur due to the order of the method that exposes. The users in group one was submitted through the VR solution of detecting the hands only using the LMC and next given the glove-based approach. For the users in group two, the glove-based solution was given before the method of LMC.

After experiencing both solutions, the participants were given a survey containing 9 Likert scale questions to collect the feedback. Ordering of the Likert scale is similar as seen in Table I. The questions provided in the questionnaire can be found in Table III.

TABLE III

QUESTIONS USED FOR THE EVALUATION OF THE GLOVE-BASED SOLUTION

\begin{tabular}{|l|l|}
\hline & Questions \\
\hline Q1 & It was difficult to wear the glove with the circuits \\
\hline Q2 & $\begin{array}{l}\text { It has been difficult to perform chest compressions with } \\
\text { the glove }\end{array}$ \\
\hline Q3 & I felt my hands were aligned with the virtual ones \\
\hline Q4 & $\begin{array}{l}\text { I experienced latency in hand movements when com- } \\
\text { pressing the chest of the patient }\end{array}$ \\
\hline Q5 & It was difficult to reach and touch the patient \\
\hline Q6 & $\begin{array}{l}\text { I felt that the occluding hand gets disappear when } \\
\text { crossing one another }\end{array}$ \\
\hline Q7 & $\begin{array}{l}\text { The overall application was up to the required realism } \\
\text { level }\end{array}$ \\
\hline Q8 & $\begin{array}{l}\text { The glove has improved the realistic level of the } \\
\text { Application }\end{array}$ \\
\hline Q9 & $\begin{array}{l}\text { I would like to use hand detection glove in the VR } \\
\text { based CPR application. }\end{array}$ \\
\hline
\end{tabular}

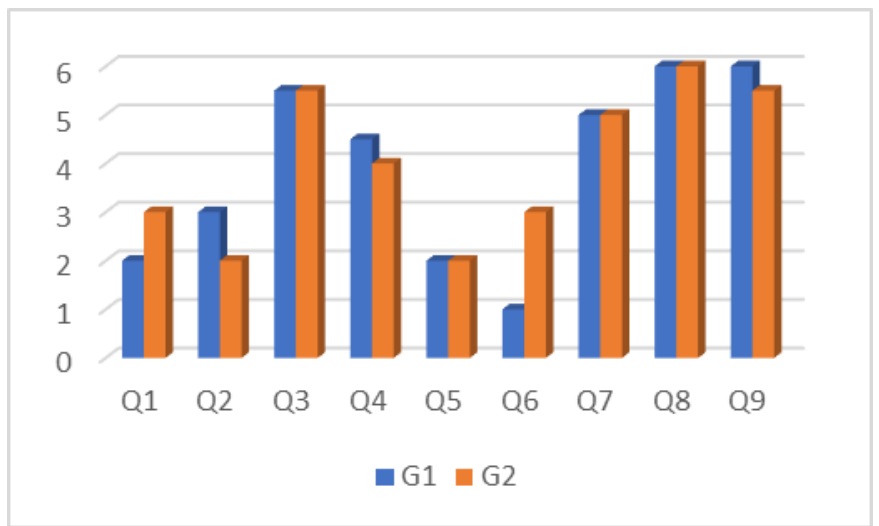

Fig. 11 Results of the User-Based Evaluation for Glove Acceptability

The comparison between $\mathrm{G} 1$ and $\mathrm{G} 2$ can be seen in figure 11. The most noticeable difference can be observed in the opinions gained for the statement Q6.

The spread of opinion for Q6 among G1 (IQR=1) and G2 $(\mathrm{IQR}=2.75)$ can be explained by some of the users marking in 
agreeing that the occluding hand gets disappears while the majority disagree on the statement. When considering the Mode values obtained for the groups G1 (=1) and G2 (=2), both are aligned with the direction of agreeing.

The opinion for Q8 as the spread among G1 (IQR=1.75) is larger compared to $\mathrm{G} 2$ (IQR=0.75), as some participants have marked disagreeing with the statement "The glove improved the realistic level of the application". But both groups can be observed with similar values for Mode $(=6)$ and Median $(=$ $6)$.

Additionally, there are some other distinctions of the opinions of two user groups according to the graph in figure 11. Considering the overall results, responses given for Q8 and Q9 are leaned towards the agreed end which infers that the users have agreed to the idea of incorporating a hand detection glove for the VR based CPR training application to improve the realistic level.

For the comparison of G1 and G2, Fisher's exact test has been used as it compares proportions and thus gives a comparison of the distribution of answers among the groups. In the test, the null hypothesis (H0) for each statement (Q1Q9) was that both groups make no significant difference in the agreement for that statement. And the alternative hypothesis (H1) was that there is a significant difference in the agreement for the given statement, while the statistical significance is $5 \%$.

As seen in Table IV, in each statement, P-value is greater than 0.05 . Thus, we cannot reject the null hypothesis which explains that there is no significant difference when a group either uses the only LMC for hand tracking first or the glovebased approach first in the evaluation. Therefore, it can be state that the order of hand tracking methodology exposed by the users do not make any vital impact on the evaluation.

TABLE IV

P-VALUES For Fisher ExACt TEST RUN ON STATEMENTS AMONG USER GROUPS G1 AND G2

\begin{tabular}{|c|l|}
\hline Statement & \multicolumn{1}{|c|}{ P-value } \\
\hline Q1 & 0.8483 \\
\hline Q2 & 0.7731 \\
\hline Q3 & 0.6356 \\
\hline Q4 & 0.5383 \\
\hline Q5 & 0.6843 \\
\hline Q6 & 0.0604 \\
\hline Q7 & 0.1 \\
\hline Q8 & 0.1125 \\
\hline Q9 & 0.1125 \\
\hline
\end{tabular}

\section{CONCLUSION AND FUTURE WORK}

The two main contributions of this study have been the development of a VR application as an affordable solution for CPR training and the implementation of a wearable glove to track the occluding hand while performing chest compressions. Carrying out implementation using off-theshelf hardware has been considered as a way of cost reduction [23], being technology becomes widely available. The opportunity of using free development software or at least the software that has a free community version cut down the licensing fees that can be also contributed to the costs. Because of this utilization of customized, cost effective setup, the proposed application is affordable to the medical sector of developing countries such as Sri Lanka.

The VR application proposed in this paper has been evaluated with two groups of users categorized by the level of proficiency in CPR. Both groups have responded favorably towards integrating VR into CPR training. The attitude of expert users expresses that they see a benefit of using VR for CPR training over the use of mechanical manikin. The positive feedback of the novice users inferred that even the users with no prior experience can use this application to gain CPR training.

The position accuracy of the glove has been assessed by comparing the $3 \mathrm{D}$ coordinates streamed out from the circuits with the real-world coordinates. The average position error in $\mathrm{X}, \mathrm{Y}, \mathrm{Z}$ directions has concluded that the coordinates given by the system are accurate. To figure out the user acceptability of the glove, feedback of the users has been collected on the experience of using the suggested occluded hand tracking method. The majority of the participants have admitted that integrating hand detection glove, increases the realistic level of the application.

To improve the realistic experience and the ability of acquiring accurate training, while making the training application more affordable, refinements must be done on the current version of the development. For future work, replacing the mechanical manikin will be the main task, as it removes the reliance on the manikin in producing haptic and tactile feedback. Manipulating the avatar to present signs of recovery is another task to be conducted.

A suitable solution for the problem of latency in vertical hand movements also must be explored. Possibly by improving the sensor (photodiode) accuracy or some other method that can handle the latency of hand movements while not disturbing the user's experience of the simulation.

A longitudinal study must be conducted in order to evaluate the output of the entire training application. The improvements gained by the trainers in the process of performing chest compressions has to be measured over a time period since acquiring the proficiency in performing $\mathrm{CPR}$ is an incremental process.

The procedures such as Endotracheal Intubation and Defibrillation can also be integrated into the solution. Intubation and Defibrillation are also skills that can be added as these are essential for target users, which is emergency medical personnel.

\section{REFERENCES}

[1] C. A. Bon, "Cardiopulmonary resuscitation (cpr)." [Online]. Available: https://emedicine.medscape.com/article/1344081-overview

[2] J. Soar, J. P. Nolan, B. W.Bottiger, K. Sunde, and C. D.Deakin, "European resuscitation council guidelines for resuscitation 2015." [Online]. Available:

https://www.resuscitationjournal.com/article/S0300-9572(15)00328-7

[3] A. Kotranza and B. Lok, "Virtual human + tangible interface $=$ mixed reality human an initial exploration with a virtual breast exam patient," IEEE Virtual Reality Conference, pp. 99-106, 2008.

[4] Y.Tian, S. Raghuraman, Y. Yand, X. Guo, and B.Prabhakaran, "3d immersive cardiopulmonary resuscitation (cpr) trainer," Proceedings of the 22nd ACM international conference on Multimedia, p. 749-750, November 2014.

[5] P. Khanal, A. Vankipuram, A. Ashby, M. Vankipuram, A. Gupta, D. Drumm-Gurnee, K. Josey, L. Tinker, and M. Smith, "Collaborative virtual reality based advanced cardiac life support training simulator using virtual reality principles," Journal of Biomedical Informatics, vol. 51, pp. 49 - 59, 2014. [Online]. Available:

http://www.sciencedirect.com/science/article/pii/S1532046414000902 
[6] S. U. Liyanage, L. Jayaratne, M. Wickramasinghe and A. Munasinghe, "Towards an Affordable Virtual Reality Solution for Cardiopulmonary Resuscitation Training," 2019 IEEE Conference on Virtual Reality and 3D User Interfaces (VR), Osaka, Japan, 2019, pp. 1054-1055, doi: 10.1109/VR.2019.8798159.

[7] S. Liyanage. (2018, July 25). An affordable solution for cardiopulmonary resuscitation training using virtual reality [video]. YouTube. https://www.youtube.com/watch?v=HYY8AZZnbzk

[8] D. T. Nguyen, W. Li, and P. O. Ogunbona, "Inter-occlusion reasoning for human detection based on variational mean field," Neurocomputing, vol. 110, pp. $51-61,2013$. [Online]. Available: http://www.sciencedirect.com/science/article/pii/S0925231213000039

[9] T. L. Baldi, S. Scheggi, L. Meli, M. Mohammadi, and D. Prattichizzo, "Gesto: A glove for enhanced sensing and touching based on inertial and magnetic sensors for hand tracking and cutaneous feedback," IEEE Transactions on Human-Machine Systems, vol. 47, no. 6, pp. 1066-1076, 2017.

[10] A. Clark and D. Moodley, "A system for a hand gesture-manipulated virtual reality environment," in Proceedings of the Annual Conference of the South African Institute of Computer Scientists and Information Technologists, ser. SAICSIT '16. New York, NY, USA: Association for Computing Machinery, 2016. [Online]. Available: https://doi.org/10.1145/2987491.2987511

[11] F. Mueller, D. Mehta, O. Sotnychenko, S. Sridhar, D. Casas, and C. Theobalt, "Real-time hand tracking under occlusion from an egocentric RGB-D sensor," CoRR, vol. abs/1704.02201, 2017 [Online]. Available: http://arxiv.org/abs/1704.02201

[12] N. Gavish, T. Gutierrez,' S. Webel, J. Rodr'1guez, M. Peveri, U. Bockholt, and F. Tecchia, "Evaluating virtual reality and augmented reality training for industrial maintenance and assembly tasks," Interactive Learning Environments, vol. 23, no. 6, pp. 778-798, 2015 [Online]. Available: https://doi.org/10.1080/10494820.2013.815221

[13] S. Pramanik and M. Mannivanan, "Immersive virtual reality based cpr training system," pp. 463-464, 2015.

[14] F. Semararo, A. Frisoli, M. Bergamasco, and E. L. Cerchiari, "Virtual reality enhanced mannequin (vrem) that is well received by resuscitation experts," Resuscitation, vol. 80, no. 4, pp. 489-492, 2009. [Online]. Available: http://www.sciencedirect.com/science/article/pii/S0300957209000136

[15] S. Yamamoto, K. Funahashi, and Y. Iwahori, "A study for vision based data glove considering hidden fingertip with self-occlusion," in 2012 13th ACIS International Conference on Software Engineering, Artificial Intelligence, Networking and Parallel/Distributed Computing, Aug 2012, pp. 315-320.

[16] T. L. Baldi, S. Scheggi, L. Meli, M. Mohammadi, and D. Prattichizzo, "Gesto: A glove for enhanced sensing and touching based on inertial and magnetic sensors for hand tracking and cutaneous feedback," IEEE Transactions on Human-Machine Systems, vol. 47, no. 6, pp. 1066-1076, Dec 2017.

[17] Y. Yang, D. Weng, D. Li, and H. Xun, "An improved method of pose estimation for lighthouse base station extension," vol. 17, p. 2411, 10 2017.

[18] D. R. Quinones, 〜 G. Lopes, D. Kim, C. Honnet, D. Moratal, and A. Kampff, "Hive tracker: A tiny, low-cost, and scalable device for submillimetric 3d positioning," in Proceedings of the 9th Augmented Human International Conference, ser. AH '18. New York, NY, USA: ACM, 2018, pp. 9:1-9:8. [Online]. Available: http://doi.acm.org/10.1145/3174910.3174935

[19] M. S. Ibrahim, A. A. Badr, M. R. Abdallah, and I. F. Eissa, "Bounding box object localization based on image superpixelization," Procedia Computer Science, vol. 13, pp. 108-119, 2012, proceedings of the International Neural Network Society Winter Conference (INNS-WC2012). [Online]. Available: http://www.sciencedirect.com/science/article/pii/S1877050912007260

[20] "An arduino library to interface the avia semiconductor hx711 24-bit analog-to-digital converter (adc) for weight scales," 2017. [Online]. Available: https://github.com/bogde/HX711

[21] S. Liyanage. (2019, March 17). An affordable virtual reality solution for cardiopulmonary resuscitation training [video]. YouTube. https://www.youtube.com/watch?v=T0r7CdRLpY4

[22] "vive-diy-position-sensor," 2017. [Online]. Available: https://github.com/ashtuchkin/vive-diy-position-sensor

[23] "Htc vive reduces price by $\$ 200$, making the best virtual reality system more accessible to the mass market,", 2017. [Online]. Available: https://www.vive.com/us/newsroom/2017-08-21/ 